\title{
Learning Bayesian network structure based on the classification and regression tree Yan Sun ${ }^{1,2}$ and Yi-Yuan Tang*1,3
}

\author{
Address: ${ }^{1}$ Institute of Neuroinformatics and Department of Computer Science, Dalian University of Technology, Dalian, China, ${ }^{2}$ Computer \\ Science Department, Liaoning Normal University, Dalian, China and ${ }^{3}$ Department of Psychology, University of Oregon, Eugene, USA \\ Email: Yi-Yuan Tang* - yiyuan@uoregon.edu \\ * Corresponding author
}

from Seventeenth Annual Computational Neuroscience Meeting: CNS*2008

Portland, OR, USA. 19-24 July 2008

Published: II July 2008

BMC Neuroscience 2008, 9(Suppl I):P7 I doi: I0. I 186/I47|-2202-9-SI-P7 I

This abstract is available from: http://www.biomedcentral.com/I47I-2202/9/SI/P7 I

(C) 2008 Sun and Tang; licensee BioMed Central Ltd.

Most methods in neuroimaging studies are based on Voxel-based morphometry (VBM) that uses a mass univariate approach to find regional morphological changes and to obviate the need for specification of ROIs. Bayesian network methods can capture multivariate and nonlinear probabilistic dependency relationships between the variables in biological experiments $[1,2]$. We applied such procedures to describe the dependency relationship among the behavioral, clinical and brain structural variables in a project for diagnosing the early stages of Alzheimer Disease $[3,4]$. First, we worked with the clinical psychologists to perform MMSE (mini-mental state examination), CDR (Clinical Dementia Rating scale), ANT (Attendant Networks Test) and STM (Short-term Memory test) for outpatients at XinHuan Hospital of Dalian University. Second, physicians interviewed each outpatient subject to get full clinical information, and then selected twenty-five potential MCI (Mild Cognitive Impairment) patients based on their clinical experiences. Then we collected MR imaging data to get the structural data for 8 regions (left/right hippocampus, left/right thalamus, left/right entorhinal and left/right amygdala) based on the previous literature. We segmented and registered data to the MNI template for imaging data. Lastly, we integrated all the behavioral, clinical and brain structural data into our proposed learning method for further diagnosis.

In order to overcome limitations of the ordinary algorithm that imposes a previous ordering on the domain attributes that restricts the number of Bayesian structures to be learned, in this paper, we present a novel structure learning method to construct the Bayesian network from these data, and the method need not prescribe a previous sequence. Using the proposed method, we first constructed a classification and regression tree to define conditional probability distributions. Then, we used the K2 algorithm to learn a Bayesian network structure from the data. Our results indicated that the MCI was mainly dependent on the hippocampus, thalamus and STM score. Although the proposed method slightly sacrificed performance efficiency, it still gave significant improvements in accuracy. In addition, not only can the method do feature selection, but it also can handle discrete and continuous inputs. In the future, we will extend the algorithm to handle missing data and hidden variables [5].

\section{Acknowledgements}

This work was supported in part by National Natural Science Foundation of China Grant 30670699, Ministry of Education Grant NCET-06-0277 and 021010.

\section{References}

I. Chen R, Herskovits EH: Network analysis of mild cognitive impairment. Neurolmage 2006, 29: I252-I259.

2. Herskovits EH, Gerring JP: Application of a data-mining method based on Bayesian networks to lesion-deficit analysis. Neurolmage 2003, 19:1664-1673.

3. Antonucci A, Zaffalon M: Fast algorithms for robust classification with Bayesian nets. International Journal of Approximate Reasoning 2007, 44:200-223.

4. Cruz-Ramirez N, Acosta-Mesa HG, Carrillo-Calvet H, Alonso NavaFernandez L, Barrientos-Martinez RE: Diagnosis of breast cancer using Bayesian networks: A case study. Comput Biol Med 2007, 37: 1553-1564.

5. Eaton D, Murphy K: Bayesian structure learning using dynamic programming and MCMC. The 23rd Conference on Uncertainty in 
Artificial Intelligence 2007 [http://www.cs.ubc.ca/ murphyk/Papers/ eaton-uai07.pdf].

Publish with Bio Med Central and every scientist can read your work free of charge

"BioMed Central will be the most significant development for disseminating the results of biomedical research in our lifetime. " Sir Paul Nurse, Cancer Research UK

Your research papers will be:

- available free of charge to the entire biomedical community

- peer reviewed and published immediately upon acceptance

- cited in PubMed and archived on PubMed Central

- yours - you keep the copyright

Submit your manuscript here:

http://www.biomedcentral.com/info/publishing_adv.asp 